\title{
Ortho versus Meta Chlorophenyl-2,3-Benzodiazepine Analogues; Synthesis, Molecular Modeling, and Biological Activity as AMPAR Antagonists.
}

Mohammad Qneibi ${ }^{1, *}$, Nidal Jaradat ${ }^{2}$, Mohammed Hawash ${ }^{2}$, Abdurrahman Olgac ${ }^{3}$, Nour Emwas $^{1}$

${ }^{1}$ Department of Biomedical Sciences, Faculty of Medicine and Health Sciences, An-Najah National University, Nablus, Palestine.

${ }^{2}$ Department of Pharmacy, Faculty of Medicine and Health Sciences, An-Najah National University, Nablus, Palestine.

${ }^{3}$ Department of Pharmaceutical Chemistry, Faculty of Pharmacy, Gazi University, Yenimahalle, Ankara, 06330, Turkey.

Correspondence: mqneibi@najah.edu; Tel.: +972-545-975-016

\section{Table of Contents}

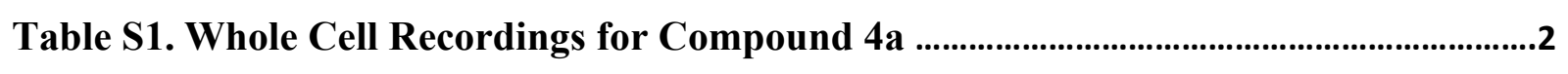

Table S2. Whole Cell Recordings for Compound 4b ……………………………........................

Figure S1. HPLC results of compound 4a ………........................................................................4

Figure S2. HPLC results of compound 4b ....................................................................................

Figure S3. Dose Response Curve for compound 4a …..................................................................6

Figure S4. Dose Response Curve for compound 4b ....................................................................... 


\begin{tabular}{|c|c|c|c|c|c|}
\hline $\begin{array}{c}\text { Receptor } \\
\text { Name/Compounds } \\
\text { abbreviation }\end{array}$ & $\begin{array}{c}\text { GluA1 } \\
\text { (Glutamate } \\
\text { Alone) }\end{array}$ & $4 a$ & $\begin{array}{l}\text { Applying Glutamate } \\
\text { Alone After 4a }\end{array}$ & $\mathbf{N}$ & $\mathbf{A} / \mathbf{A}_{\mathbf{I}}$ \\
\hline $\begin{array}{l}\text { Amplitude } \\
\qquad(\mathrm{pA})\end{array}$ & $967 \pm 32$ & $144 \pm 21^{* * *}$ & $971 \pm 27$ & 6 & $6.7 \pm 0.1$ \\
\hline $\begin{array}{c}\text { t deact } \\
(\mathrm{ms})\end{array}$ & $2.1 \pm 0.1$ & $2.3 \pm 0.2^{\mathrm{ns}}$ & $\mathrm{N} / \mathrm{R}$ & 6 & $\mathrm{~N} / \mathrm{R}$ \\
\hline $\begin{array}{l}\text { t des } \\
\text { (ms) }\end{array}$ & $2.6 \pm 0.1$ & $4.2 \pm 0.2^{* * *}$ & $\mathrm{~N} / \mathrm{R}$ & 6 & $\mathrm{~N} / \mathrm{R}$ \\
\hline $\begin{array}{c}\text { Receptor } \\
\text { Name/Compounds } \\
\text { abbreviation }\end{array}$ & $\begin{array}{c}\text { GluA1/2 } \\
\text { (Glutamate } \\
\text { Alone) }\end{array}$ & $4 a$ & $\begin{array}{l}\text { Applying Glutamate } \\
\text { Alone After 4a }\end{array}$ & $\mathbf{N}$ & $\mathbf{A} / \mathbf{A}_{\mathbf{I}}$ \\
\hline $\begin{array}{l}\text { Amplitude } \\
\qquad(\mathbf{p A})\end{array}$ & $480 \pm 31$ & $87 \pm 15^{* * *}$ & $470 \pm 41$ & 7 & $5.5 \pm 0.2$ \\
\hline $\begin{array}{c}\text { t deact } \\
(\mathrm{ms})\end{array}$ & $2.6 \pm 0.2$ & $2.5 \pm 0.2^{\mathrm{ns}}$ & $\mathrm{N} / \mathrm{R}$ & 7 & $\mathrm{~N} / \mathrm{R}$ \\
\hline $\begin{array}{l}\mathrm{t} \text { des } \\
\text { (ms) }\end{array}$ & $4.9 \pm 0.1$ & $6.3 \pm 0.2^{* * *}$ & $\mathrm{~N} / \mathrm{R}$ & 7 & $\mathrm{~N} / \mathrm{R}$ \\
\hline $\begin{array}{c}\text { Receptor } \\
\text { Name/Compounds } \\
\text { abbreviation }\end{array}$ & $\begin{array}{c}\text { GluA2 } \\
\text { (Glutamate } \\
\text { Alone) }\end{array}$ & $4 a$ & $\begin{array}{l}\text { Applying Glutamate } \\
\text { Alone After 4a }\end{array}$ & $\mathbf{N}$ & $\mathbf{A} / \mathbf{A}_{\mathbf{I}}$ \\
\hline $\begin{array}{l}\text { Amplitude } \\
\qquad(\mathbf{p A})\end{array}$ & $1290 \pm 62$ & $208 \pm 28^{* * *}$ & $1296 \pm 55$ & 6 & $6.2 \pm 0.1$ \\
\hline $\begin{array}{l}\text { t deact } \\
(\mathrm{ms})\end{array}$ & $2.3 \pm 0.1$ & $2.5 \pm 0.1^{\mathrm{ns}}$ & $\mathrm{N} / \mathrm{R}$ & 6 & $\mathrm{~N} / \mathrm{R}$ \\
\hline $\begin{array}{l}\mathrm{t} \text { des } \\
(\mathrm{ms})\end{array}$ & $2.8 \pm 0.1$ & $4.6 \pm 0.2^{* * *}$ & $\mathrm{~N} / \mathrm{R}$ & 6 & $\mathrm{~N} / \mathrm{R}$ \\
\hline
\end{tabular}

Table S1. Whole Cell Recordings for Compound 4a 
Table S2. Whole Cell Recordings for Compound 4b

\begin{tabular}{|c|c|c|c|c|c|}
\hline $\begin{array}{c}\text { Receptor } \\
\text { Name/Compounds } \\
\text { abbreviation }\end{array}$ & $\begin{array}{c}\text { GluA1 } \\
\text { (Glutamate } \\
\text { Alone) }\end{array}$ & $4 b$ & $\begin{array}{c}\text { Applying } \\
\text { Glutamate Alone } \\
\text { After 4b }\end{array}$ & $\mathbf{n}$ & $\mathbf{A} / \mathbf{A}_{\mathbf{I}}$ \\
\hline $\begin{array}{l}\text { Amplitude } \\
\qquad(\mathrm{pA})\end{array}$ & $930 \pm 37$ & $715 \pm 27^{\mathrm{ns}}$ & $922 \pm 42$ & 6 & $1.4 \pm 0.1$ \\
\hline $\begin{array}{l}\text { t deact } \\
(\mathrm{ms})\end{array}$ & $\mathrm{N} / \mathrm{R}$ & $2.2 \pm 0.1^{\mathrm{ns}}$ & $\mathrm{N} / \mathrm{R}$ & 6 & $\mathrm{~N} / \mathrm{R}$ \\
\hline $\begin{array}{l}\text { t des } \\
(\mathrm{ms})\end{array}$ & $\mathrm{N} / \mathrm{R}$ & $2.8 \pm 0.1^{\mathrm{ns}}$ & $\mathrm{N} / \mathrm{R}$ & 6 & $\mathrm{~N} / \mathrm{R}$ \\
\hline $\begin{array}{c}\text { Receptor } \\
\text { Name/Compounds } \\
\text { abbreviation }\end{array}$ & $\begin{array}{c}\text { GluA1/2 } \\
\text { (Glutamate } \\
\text { Alone) }\end{array}$ & $4 b$ & $\begin{array}{c}\text { Applying } \\
\text { Glutamate Alone } \\
\text { After } 4 b\end{array}$ & $\mathbf{n}$ & $\mathbf{A} / \mathbf{A}_{\mathbf{I}}$ \\
\hline $\begin{array}{l}\text { Amplitude } \\
\qquad \text { (pA) }\end{array}$ & $530 \pm 38$ & $481 \pm 22^{\mathrm{ns}}$ & $533 \pm 37$ & 8 & $1.1 \pm 0.2$ \\
\hline $\begin{array}{c}\text { t deact } \\
(\mathrm{ms})\end{array}$ & $\mathrm{N} / \mathrm{R}$ & $2.5 \pm 0.3^{\mathrm{ns}}$ & $\mathrm{N} / \mathrm{R}$ & 8 & $\mathrm{~N} / \mathrm{R}$ \\
\hline $\begin{array}{l}\text { t des } \\
(\mathrm{ms})\end{array}$ & $\mathrm{N} / \mathrm{R}$ & $5.0 \pm 0.2^{\mathrm{ns}}$ & $\mathrm{N} / \mathrm{R}$ & 8 & $\mathrm{~N} / \mathrm{R}$ \\
\hline $\begin{array}{c}\text { Receptor } \\
\text { Name/Compounds } \\
\text { abbreviation }\end{array}$ & $\begin{array}{c}\text { GluA2 } \\
\text { (Glutamate } \\
\text { Alone) }\end{array}$ & $4 b$ & $\begin{array}{c}\text { Applying } \\
\text { Glutamate Alone } \\
\text { After } 4 \mathrm{~b}\end{array}$ & $\mathbf{n}$ & $\mathbf{A} / \mathbf{A}_{\mathbf{I}}$ \\
\hline $\begin{array}{l}\text { Amplitude } \\
\qquad(\mathrm{pA})\end{array}$ & $1102 \pm 56$ & $847 \pm 35^{\text {ns }}$ & $787 \pm 43$ & 6 & $1.3 \pm 0.1$ \\
\hline $\begin{array}{l}\text { t deact } \\
(\mathrm{ms})\end{array}$ & $\mathrm{N} / \mathrm{R}$ & $2.4 \pm 0.1^{\mathrm{ns}}$ & $\mathrm{N} / \mathrm{R}$ & 6 & $\mathrm{~N} / \mathrm{R}$ \\
\hline $\begin{array}{l}\text { t des } \\
(\mathrm{ms})\end{array}$ & $\mathrm{N} / \mathrm{R}$ & $2.9 \pm 0.2^{\mathrm{ns}}$ & $\mathrm{N} / \mathrm{R}$ & 6 & $\mathrm{~N} / \mathrm{R}$ \\
\hline
\end{tabular}


$\underline{\text { HPLC results : }}$

Figure S1. HPLC results of compound 4a

purity $98 \%$ 


\section{==== Shimadzu LCMSsolution Analysis Report ====}

$\begin{array}{ll}\text { Acquired by } & \text { :Admin } \\ \text { Sample Name } & : \text { MMH510 } \\ \text { Sample ID } & \vdots \\ \text { Vail \# } & : 32 \\ \text { Injection Volume } & : 0.3 \mathrm{uL} \\ \text { Data File Name } & : \text { MMH510_158.Icd } \\ \text { Method File Name } & : \text { genel.Icm } \\ \text { Batch File Name } & \vdots \text { batch.Icb } \\ \text { Report File Name } & : \text { DefaultLCMS.Icr } \\ \text { Data Acquired } & : 28.12 .201814: 14: 39 \\ \text { Data Processed } & : 30.12 .201911: 16: 28\end{array}$

\section{$<$ Chromatogram $>$}

Segment\#1 $(\times 100,000)$
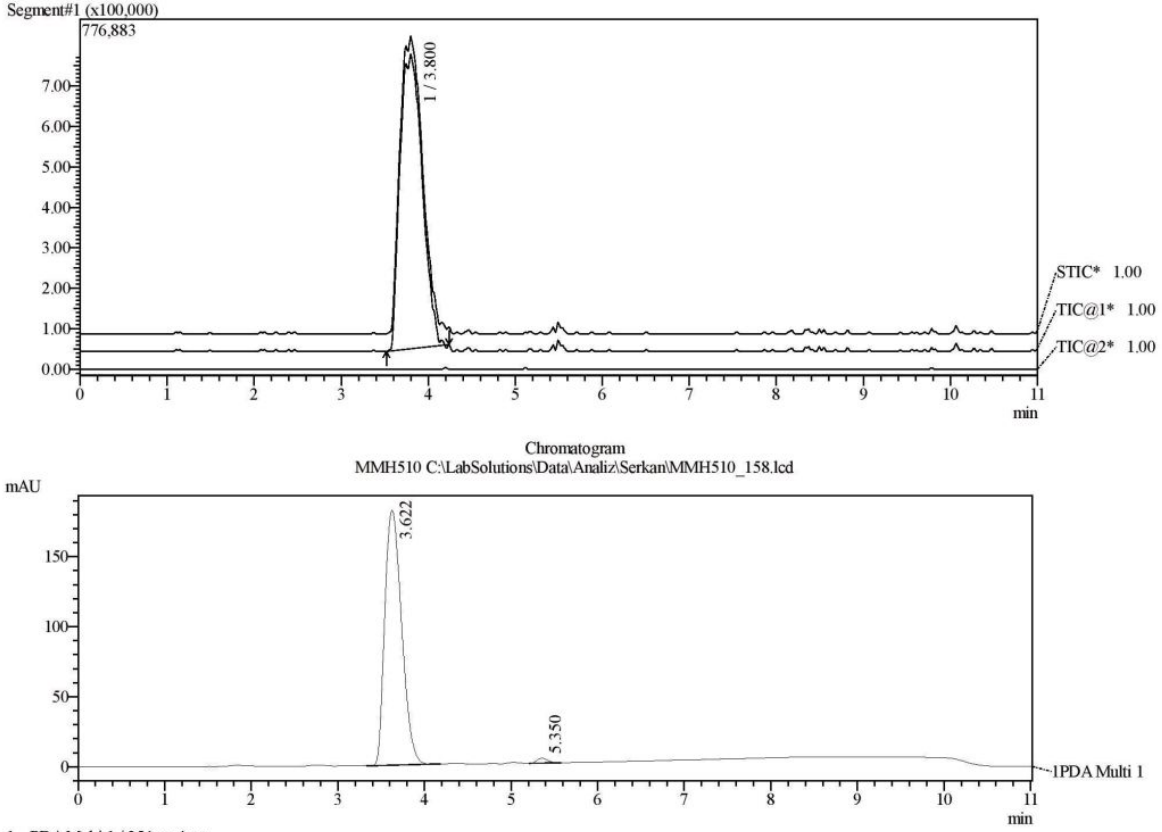

1 PDAMulti $1 / 254 \mathrm{~nm} 4 \mathrm{~nm}$
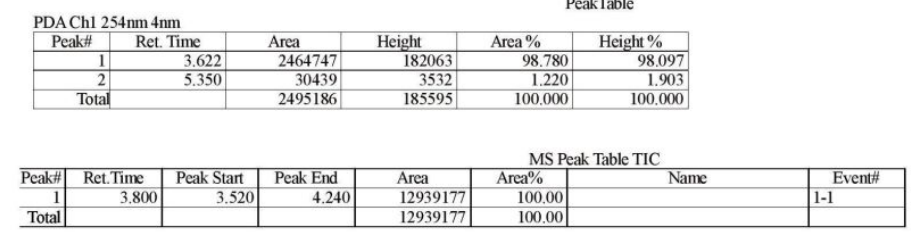

\section{Figure S2. HPLC results of compound 4b}

99\% purity 
==== Shimadzu LCMSsolution Analysis Report ====

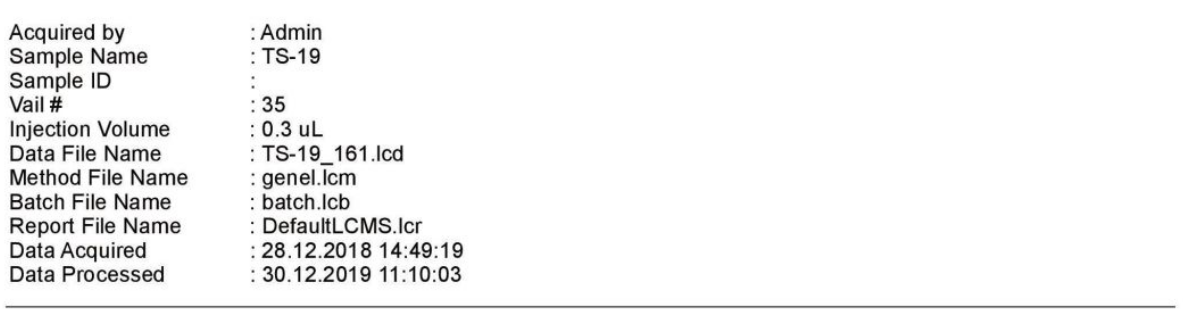

$<$ Chromatogram>

Segment\#1 (x1,000,000)
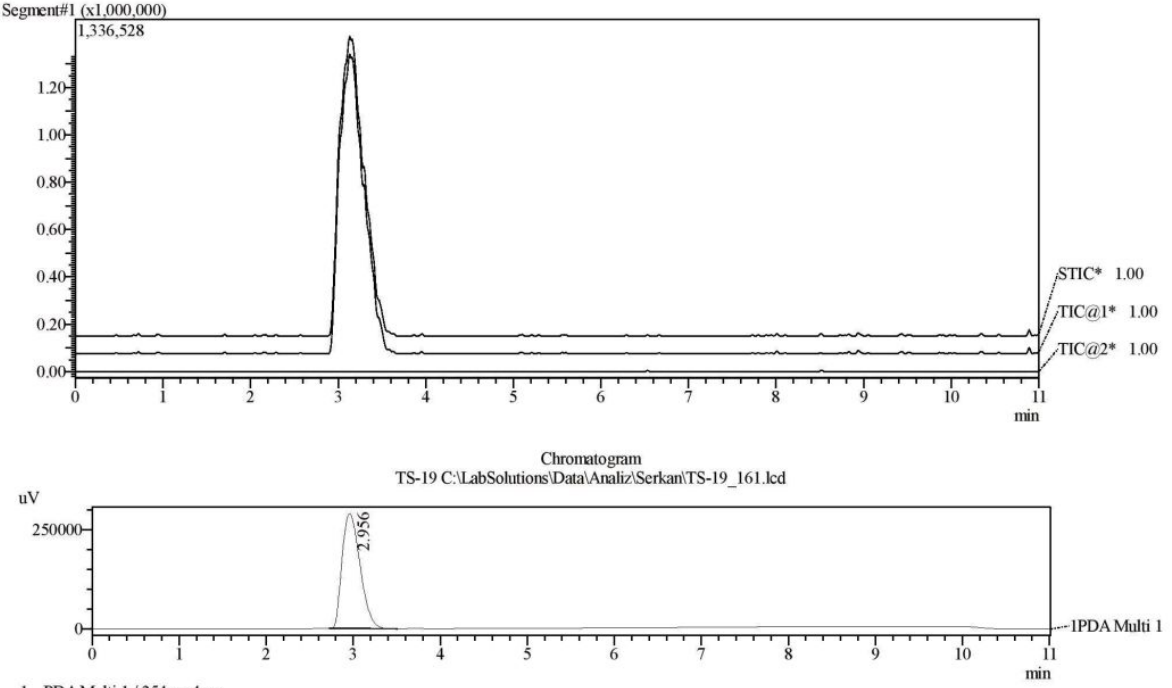

1 PDAMulti $1 / 254 \mathrm{~nm} 4 \mathrm{~nm}$

PeakTable

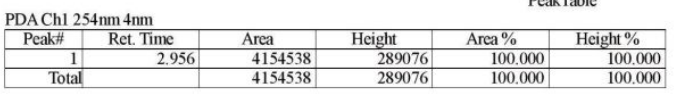

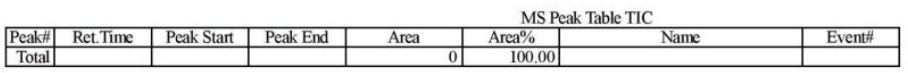

Figure S3. Dose Response Curve for compound 4a 


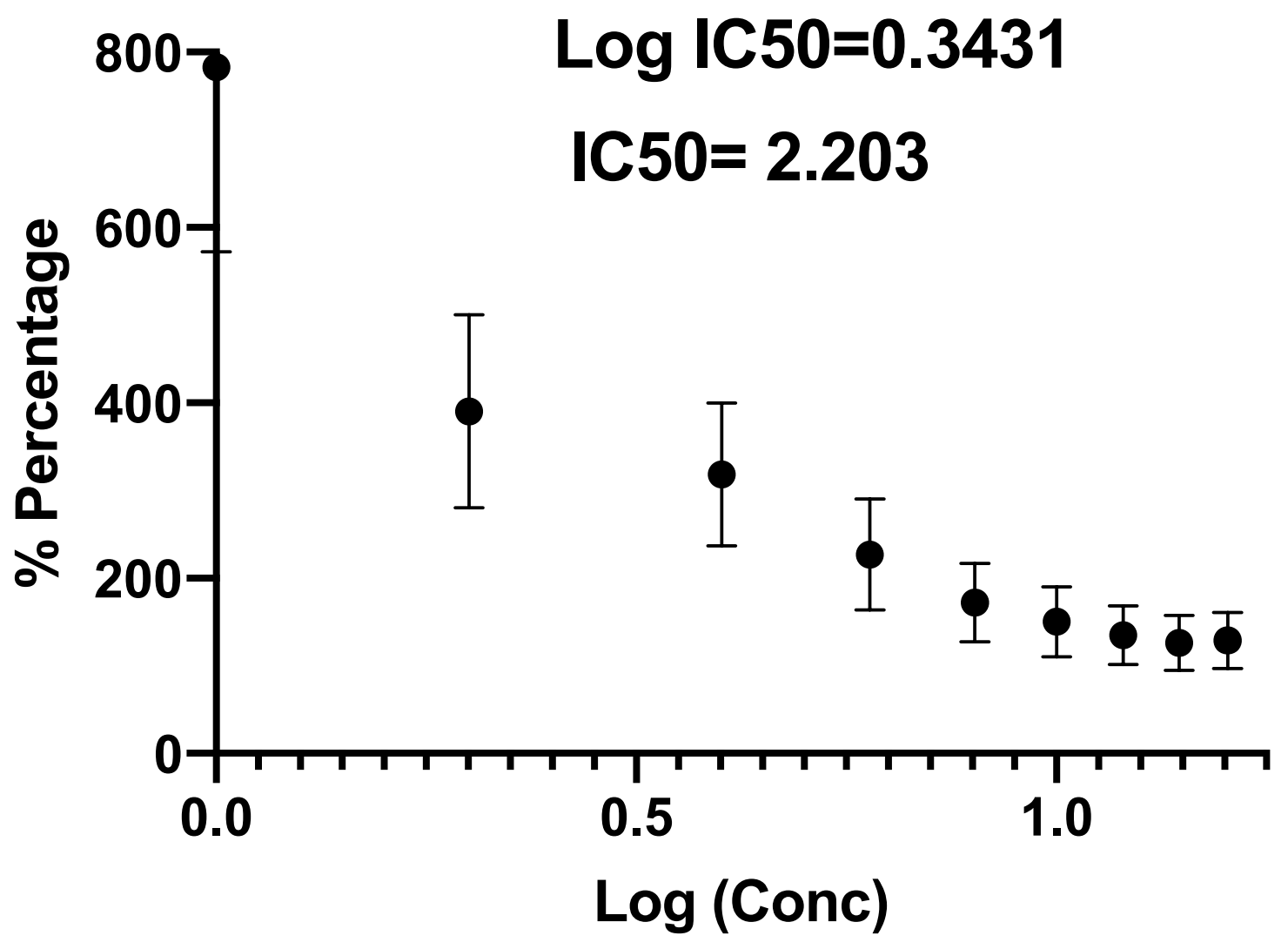

Figure S3: Dose Response Curve on GluA1 AMPAR subunit.

Determination of IC50 values for 4a on whole-cell amplitude in HEK239 cell. Each concentration was applied to the cell using the $500 \mathrm{~ms}$ protocol and then washed out for the $20 \mathrm{~s}$. After several washout intervals, glutamate alone $(10 \mathrm{mM})$ was applied, and the instantaneous evoked current was measured. Each data point represents the average of the values obtained from 3 to 4 independent experiments. Error bars represent the standard error of the means. IC50 values were determined using GraphPad Prism. 


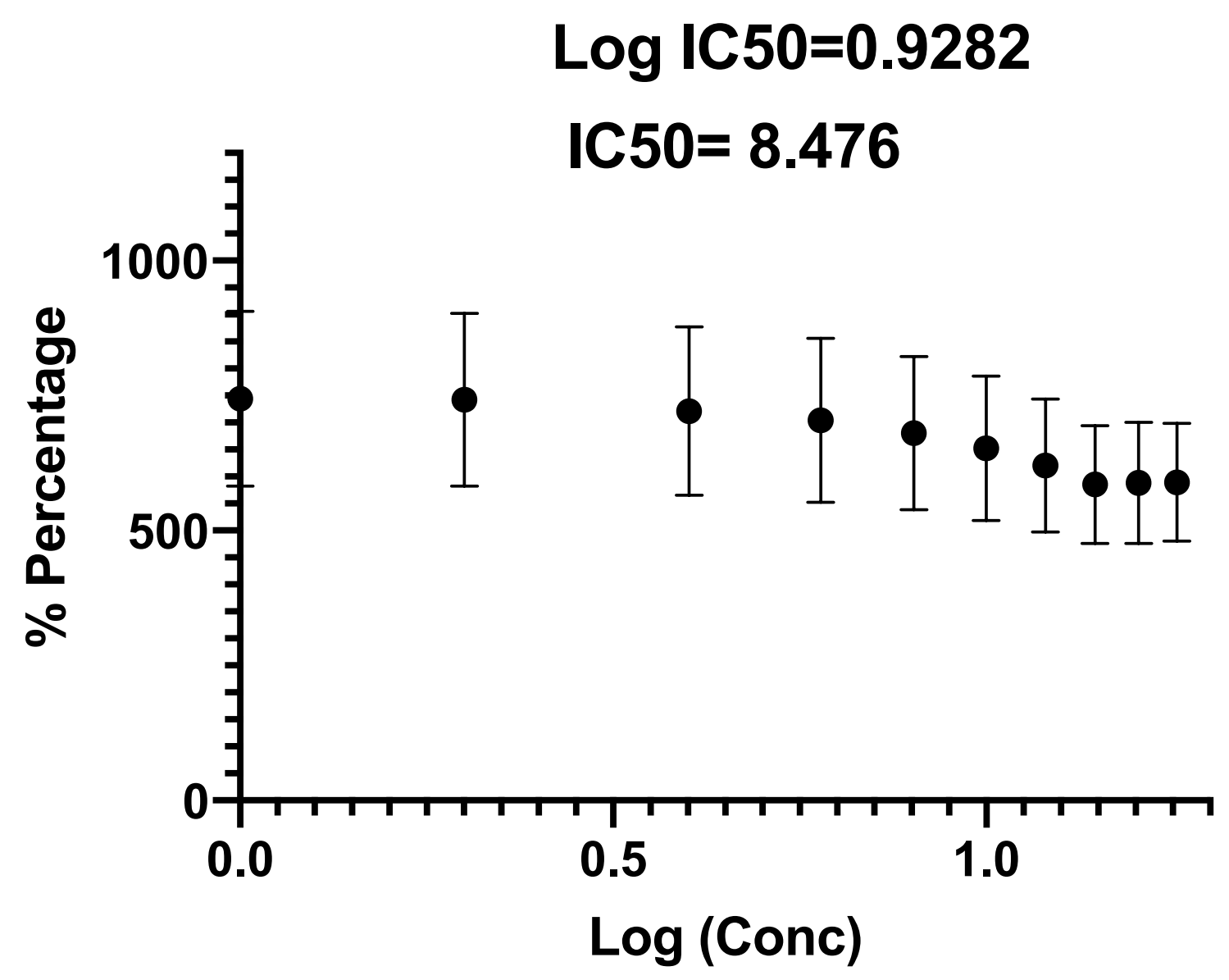

Figure S4: Dose Response Curve of GluA1 AMPAR subunit.

Determination of IC50 values for $\mathbf{4 b}$ on whole-cell amplitude in HEK239 cell lines. Each concentration was applied to the cell using the $500 \mathrm{~ms}$ protocol and then washed out for the $20 \mathrm{~s}$. After several washout intervals, glutamate alone $(10 \mathrm{mM})$ was applied, and the instantaneous evoked current was measured. Each data point represents the average of the values obtained from 3 to 4 independent experiments. Error bars represent the standard error of the means. IC50 values were determined using GraphPad Prism. 